Résumés des conférences et travaux

\title{
Littérature architectonique, philologie du texte et histoire de l'architecture
}

Francesco Paolo Di Teodoro

\section{OpenEdition}

\section{Journals}

Édition électronique

URL : https://journals.openedition.org/ashp/1323

DOI : 10.4000/ashp.1323

ISSN : 1969-6310

Éditeur

Publications de l'École Pratique des Hautes Études

Édition imprimée

Date de publication : 1 octobre 2012

Pagination : 209-214

ISSN : 0766-0677

Référence électronique

Francesco Paolo Di Teodoro, «Littérature architectonique, philologie du texte et histoire de l'architecture », Annuaire de l'École pratique des hautes études (EPHE), Section des sciences historiques et philologiques [En ligne], 143 | 2012, mis en ligne le 03 octobre 2012, consulté le 03 août 2021. URL : http://journals.openedition.org/ashp/1323 ; DOI : https://doi.org/10.4000/ashp.1323 


\title{
LITTÉRATURE ARCHITECTONIQUE, PHILOLOGIE DU TEXTE ET HISTOIRE DE L'ARCHITECTURE
}

\author{
Conférences de M. Francesco Paolo Di Teodoro, \\ École polytechnique de Turin, \\ directeur d'études invité
}

Les conférences suivent le fil conducteur de la philologie : la première est dédiée à Piero della Francesca et sa théorie du dessin en projection orthogonale; les deux suivantes abordent Vitruve et son De architectura (conférences n ${ }^{\text {os }}$ II et III); enfin la dernière conférence, en proposant un examen ultérieur de ces deux auteurs, constitue le point de convergence de l'ensemble des sujets abordés au cours des interventions.

\section{La "philologie du dessin géométrique-historique» et l'édition des textes : la Descriptio urbis Romae de Leon Battista Alberti et le Libellus de quinque corporibus regularibus de Piero della Francesca}

Dans le domaine des travaux spécialisés, peu d'espace a été alloué au dessin géométrique ancien ou historique, car on a tendance, lorsqu'un texte accompagne des dessins, à considérer ces derniers comme de simples (voire d'accessoires) véhicules conventionnels de la pensée géométrique abstraite. Ce type de dessin a ainsi très peu suscité la curiosité des connaisseurs.

La philologie du dessin géométrique-historique naît à l'occasion de la préparation de l'édition critique du Libellus de quinque corporibus regularibus de Piero della Francesca (publiée en 1995). Le Libellus est un traité de géométrie sur les corps réguliers; il est divisé en trois livres auxquels Piero ajoute à la suite un quatrième volume, le De corporibus irregularibus, qui traite des corps irréguliers et d'Archimède. Le Libellus nous est parvenu en un seul exemplaire en parchemin, le manuscrit Vat. Urb. Lat. 632, réalisé par un copiste, avec des corrections, des adjonctions et 174 dessins de la main de Piero della Francesca.

La presque totalité des dessins concernant les figures dans l'espace sont en axonométrie (projection oblique) ou en projection orthogonale, ce qui ne doit pas nous étonner pour un traité de géométrie qui requiert la possibilité de déduire immédiatement du dessin les dimensions exactes des figures dans l'espace. Le recours à la double projection orthogonale (en plan et en élévation), où les deux figures sont à la même échelle, revêt un intérêt particulier : c'est un procédé que Piero della Francesca a déjà employé dans le troisième livre de son De prospectiva pingendi. Cette méthode est propre au dessin architecturale en plan, élévation et coupe que Raphaël aurait codifiées en 1519 dans la présumée Lettre à Léon X (cf. conférence $\mathrm{n}^{\circ}$ IV).

Comme il n'existait ni précédents à imiter ni tradition soumise au contrôle du temps, la seule manière d'agir était de se faire éditeurs du texte graphique en empruntant les 
méthodes et les principes de la critique du texte, « science » qui s'est perfectionnée en l'espace d'un siècle grâce à l'exercice et au débat théorique, et dont la méthode s'adapte selon moi parfaitement aux textes graphiques.

En adaptant opportunément aux dessins l'ensemble des opérations qui constituent la critique textuelle, on peut disposer d'un instrument qui permette de rétablir le texte graphique selon les intentions originales de l'auteur, dans le respect d'une culture mathématique et figurative dont on ne doit ni altérer ni actualiser les conventions représentatives.

L'édition critique des dessins des traités théoriques de Piero della Francesca est fondée sur l'exécution d'un double dessein : un dessin diplomatique et un dessin critique (ou critique-interprétatif).

Si le Libellus a constitué le but de la construction de la philologie du dessin géométrique historique, la Descriptio urbis Romae de Leon Battista Alberti, un petit traité de topographie sui generis, nous a donné la possibilité d'approfondir la théorie, en sa possible extension dans le cas d'un texte transmis par plusieurs témoins.

La méthode du relief urbain « ex mathematicis instrumentis » ouvre la voie aux expériences du début du $\mathrm{XVI}^{\mathrm{e}}$ siècle qui auront recours au compas magnétique afin de mieux décrire les murs, les rues, les fleuves, les édifices et dont l'aboutissement le plus remarquable est sans doute le relevé de Rome dont Raphaël traite dans la Lettre à Leon X.

\section{Bibliographie}

Edizione Nazionale delle opere di Piero della Francesca, I. Libellus de quinque corporibus regularibus, vol. II : I disegni, F. P. Di Teodoro (éd.), Florence, Giunti, 1995.

F. P. Di Teodoro, «Per una filologia del disegno geometrico », dans M. Dalai Emiliani et V. Curzi (éd.), Piero della Francesca tra arte e scienza. Atti del Convegno internazionale, Venise, Marsilio, 1996, p. 239-251.

Id., « La Descriptio Urbis Romae », dans F. P. Fiore (éd.), La Roma di Leon Battista Alberti. Umanisti, architetti e artisti alla scoperta dell'antico nella città del Quattrocento. Catalogo della mostra, Milan, Skira, 2005, p. 176-179;

Id., compte rendu : Leonis Baptistce Alberti Descriptio vrbis Romce..., Jean-Yves Boriaud et Francesco Furlan (éd.), Paris, S.I.L.B.A. - Florence, Olschki, 2005, 154 p. [+ 8], 14 fig., 10 tabl., dans Albertiana, IX (2006), p. 243-257.

II. Autour d'un manuscrit de l'Architecture ou Art de bien bastir :

\section{le De architectura de Vitruve traduit par Jean Martin}

L'Architecture ou Art de bien bastir, publié à Paris en 1547 par Jacques Gazeau, s'inscrit dans le contexte du renouveau architectural français des années 1545-1550, qui correspond à l'apparition d'une architecture utilisant les ordres antiques. L'ouvrage est remarquable par ses planches qui sont en partie empruntées à l'édition vénitienne de Vitruve due à Fra Giocondo (1511), à l'édition de Cesare Cesariano (1521), et aux Secondo Libro (Paris, 1545) et Quarto Libro (Venise, 1537) de Serlio. Une autre partie des planches est en revanche dessinée et gravée par le sculpteur et architecte Jean Goujon, qui en témoigne dans sa lettre Aux lecteurs à la fin du livre (c. D iij r).

On connait seulement deux manuscrits de la traduction de Martin. Le premier, découvert et annoncé par Toshinori Uetani en 2004, a été étudié par Uetani et Henri 
Zerner en 2005 (Revue de l'Art, 149, p. 27-32). Le manuscrit, qui contient seulement le premier livre et fut probablement confectionné à Paris en 1544-1545, est conservé à Paris à la Bibliothèque nationale de France, ms. français 12338.

Le deuxième manuscrit est inconnu. Conservé à la Bibliothèque nationale universitaire de Turin (L2-I-1), ses pages comportent 133 dessins. Un incendie en 1904 l'a beaucoup endommagé. Il fut restauré en 1986, et depuis quelques années seulement on l'a restitué à la consultation et aux études. Aujourd'hui, le manuscrit se compose de 87 feuillets détachés, la reliure ayant été perdue, elle aussi, pendant l'incendie.

On peut démontrer que le manuscrit de Turin succède à la version imprimée, dont il constitue une copie (le texte surtout). De plus, la scénographie (Vitr. I 2 2) est tirée d'une gravure de Jacques Androuet du Cerceau, une grande composition d'architecture imprimée à Orléans et datée de 1551.

Le Vitruve de Turin est caractérisé par de superbes dessins à la pierre noire et à la plume agrémentés d'un lavis à l'encre brune et grise. La très bonne qualité qui caractérise de nombreux dessins révèle le travail d'un atelier artistique au courant des nouveautés architecturales (Serlio, Lescot, De L'Orme) - peut-être avec l'aide et la consultation de Sebastiano Serlio, de Pierre Lescot (et de Du Cerceau?) - dirigé par une figure artistique qui présente à la fois les caractéristiques de l'architecte et du peintre-sculpteur. Je propose, bien qu'avec prudence, d'identifier une telle figure, le « Maître de Turin », avec Jean Goujon.

\section{Bibliographie}

F. P. Di Teodoro, «Le texte de Vitruve dans les années 1540. Autour d'un manuscrit de l'Architecture ou Art de bien bastir : le De architectura de Vitruve traduit par Jean Martin ", dans S. Frommel, L. Baumer et F. Elsig (éd.), Les années 1540 : regards croisés sur les arts et les lettres. Actes du colloque international, Genève, 11-12 avril 2011, sous presse.

Id., "Vitruvio volgarizzato e identità locali : prime indagini », dans Architettura e identità locali. Conv. Int. di Studi, Bologna 26-27 gennaio 2011, sous presse.

\section{La traduction du De architectura de Vitruve par Fabio Calvo pour Raphaël (München, BSB, codd. It. 37 et It. $37^{\circ}$ ) : le texte, son exégèse, questions particulières}

La traduction vitruvienne de Fabio Calvo est aujourd'hui attestée par deux manuscrits gardés à la Bayerische Staatsbibliothek de Munich : Codex Italicus 37 (complet et avec presque 30 notes autographes de Raphaël) et Codex Italicus $37 a$ (qui s'arrête au premier chapitre du cinquième livre) qui fait partie du Cod. It. 37abc: un manuscrit mélangé composé au XIX ${ }^{\mathrm{e}}$ siècle par le bibliotécaire Johann Andreas Schmeller, dont le Cod. It. $37 b$ constitue l'un des trois manuscrits de la Lettre à Léon $X$ (cf. conférence $\mathrm{n}^{\mathrm{o}} \mathrm{IV}$ ). Le Cod. It. 37c est un traité épigraphique, le Fragmentum de literis, partiellement autographe, dû à Fra Giocondo et publié pour la première fois par Lucia Ciapponi en 1979.

On ne connaît pas encore le nom du scribe des deux manuscrits vitruviens et leur datation étant, elle aussi, très problématique, la question demeure ouverte.

La traduction de Fabio Calvo a été publiée en 1975 par Vincenzo Fontana et Paolo Morachiello de façon très incorrecte. Elle comporte en effet plusieurs types d'erreurs significatives, de différents niveaux de gravité, mis en évidence par la nouvelle édition 
à laquelle je travaille. Il s'agit d'homéoarchies, des erreurs optiques ou paléographiques, beaucoup d'erreurs de lecture, des erreurs relatives au système d'abréviation, des innovations au texte.

Tout en s'appuyant sur l'édition de Fra Giocondo, la traduction de Calvo se sert aussi de toutes les éditions précédentes et de quelques manuscrits. Le traducteur ne suit pas toujours le texte établi par Giocondo : il accueuille très souvent des leçons de l'editio princeps (1487-1488) et, surtout, de l'editio veneta (1497).

La traduction de Fabio Calvo en volgare témoigne en définitive d'un travail mené en s'appuyant sur une pluralité de sources manuscrites, comme une sorte de recensio partielle de témoins, surtout dans les cas où entrent en jeu des questions concernant les modules, les proportions ou tout simplement les dimensions. À ce titre, elle s'avère être un véritable collecteur de variantes dont les éditions critiques modernes ne nous aident pas à identifier les sources. Ce dont nous avons réellement besoin pour Vitruve, donc, ce n'est pas seulement d'un texte critique; c'est aussi et surtout d'une édition qui prenne en compte tous les témoins connus, avec leurs fautes et leurs variantes. Les manuscrits latins, bien évidemment, mais aussi les manuscrits en volgare, dans la mesure où ceux-ci peuvent être porteurs de leçons de l'un ou de plusieurs manuscrits latins encore inconnus ou bien aujourd'hui disparus. Nous avons besoin d'une édition qui conduise à une interprétation correcte, en particulier, de l'architecture de la Renaissance, une édition qui ne nous oblige pas à en confronter les aboutissements avec un texte qui n'existait pas. Il convient par conséquent d'établir, en collaboration avec une équipe de chercheurs, une édition informatisée.

Lorsqu'il sera possible de connaître et d'interroger le texte de tous les témoins subsistants de Vitruve, les historiens de l'art seront enfin en mesure de réviser quelques passages historico-critiques centraux concernant les théories architecturales, le « vitruvianisme » et l'« architecture de genre vitruvien », surtout aux époques qui ont placé le De architectura au centre de leur art de bâtir, lui attribuant un rôle de moteur du renouveau architectural.

\section{Bibliographie}

F. P. Di Teodoro, «Per una nuova edizione del De architectura di Vitruvio tradotto da Fabio Calvo per Raffaello : qualche nota sui primi tre libri del Cod. It. 37 della Bayerische Staatsbibliothek di Monaco », dans H. Hubach, T. Tassini, B. von Orelli-Messerli (éd.), Reibungspunkte. Ordnung und Umbruch in Architektur und Kunst. Festschrift für Hubertus Günther, Petersberg, Imhof, 2008, p. 57-62.

Id., « Per l'edizione del Vitruvio di Fabio Calvo per Raffaello », dans F. P. Di Teodoro (éd.), Saggi di letteratura architettonica, da Vitruvio a Winckelmann, I, Florence, Olschki, 2009, p. 191-206.

Id., "Spigolature dal IV Libro del Vitruvio di Fabio Calvo per Raffaello (Bayerische Staatsbibliothek Muenchen, Cod. It. 37) », dans L. Bertolini (éd.), Saggi di letteratura architettonica, da Vitruvio a Winckelmann, II, Florence, Olschki, 2009.

Id., « Glosse, interpolazioni e correzioni nel Vitruvio tradotto da Fabio Calvo (Bayerische Staatsbibliothek, Cod. It. 37) : tra lavoro d'équipe e autografi di Raffaello », dans H. Burns, F. P. Di Teodoro et G. Bacci (éd.), Saggi di letteratura architettonica, da Vitruvio a Winckelmann, III, Florence, Olschki, 2010, p. 177-196.

Id., "Quel(s) Vitruve? Le De architectura au début du XvI $\mathrm{I}^{\mathrm{e}}$ siècle à la lumière de la traduction de Fabio Calvo pour Raphaël », Albertiana, XIV (2011), p. 121-141. 


\section{Raphaël, Baldassar Castiglione et la Lettre à Léon X : le contexte, les trois manuscrits, le texte imprimé en 1733, les antiquités de Rome}

La Lettre à Léon $X$ est l'épître dédicatoire, avant son perfectionnement et son homogénéisation, qui aurait dû accompagner un relevé de la Rome antique par Raphaël.

Elle devait en outre, selon les interprétations les plus couramment acceptées, préluder à un traité d'architecture illustré par des dessins d'édifices antiques. Représentés selon une méthode rigoureuse, ces édifices auraient été figurés non pas à l'état de ruine, mais sous leur forme originelle, comme Serlio allait le faire, vingt ans plus tard, dans son Terzo Libro (1540).

De fait, la Lettre fait clairement allusion à un plan de la Ville antique : après avoir procédé à un relevé de Rome du haut de ses collines et avoir partagé la ville en quatorze regiones (comme on l'avait fait à l'époque d'Auguste), Raphaël aurait relevé le plan de tous les édifices; les plus remarquables devaient ensuite être présentés séparément selon trois «modes »: plan, élévation et coupe (en projection orthogonale). Ainsi outre les dessins des édifices exemplaires, le traité devait être aussi accompagné d'un plan ichnométrique de l'ensemble de la ville. Ce dernier aurait également mis en évidence les liens entre la Rome antique et la Rome moderne, grâce à un système de relations et de connexions permettant d'en suivre l'histoire. Seule leur interaction avec l'organisme urbain qui les accueille permet en effet aux édifices, pris séparément, d'être soustraits à une lecture anhistorique. Un traité ainsi conçu aurait constitué une innovation décisive; personne après Raphaël ne réussit à en concevoir, et encore moins à en réaliser un semblable.

Le texte connu sous la dénomination de Lettre à Léon X est attesté par quatre documents, dont trois sont manuscrits et le quatrième imprimé.

1) Un premier manuscrit, autographe de Baldassar Castiglione, est conservé à Mantoue dans les archives des comtes Castiglioni (Documenti Sciolti a, n. 12);

2) Le deuxième manuscrit, encore inédit et que je suis en train de publier, est conservé à Mantoue dans une collection privée;

3) Le troisième manuscrit est conservé à la Bayerische Staatsbibliothek de Munich et fait partie d'un recueil de pièces diverses, formant le Cod. It. 37abc. Le document qui nous intéresse, le Cod. It. 37b, occupe les folios 75 à 89 du manuscrit.

4) La quatrième source de la Lettre est constituée par le texte imprimé à Padoue en 1733, chez l'éditeur Comino à l'initiative des frères Giovanni Antonio et Gaetano Volpi, dans le corpus des Opere volgari e latine del Conte Baldessar Castiglione.

La quatrième conférence nous a donné l'occasion de traiter surtout du deuxième manuscrit inédit de la Lettre qui, même si maintenant est conservé à Mantoue dans une collection privée, a été jusqu'au XVIII ${ }^{\mathrm{e}}$ siècle conservé dans les archives Castiglioni. Il fait partie d'un recueil composé de 15 fascicules pour un total de 307 feuillets, et de 162 lettres environ, les Lettere del Conte Baldassar Castiglione cioè copie. Le verso de la couverture antérieure porte des références à un livre publié en 1581. À l'intérieur de la couverture postérieure il y a des annotations relative à la famille Castiglioni, écrites par Francesco Castiglioni qui y rappelle les dates de naissance de ses enfants de 1623 jusqu'à 1643 . Le document qui nous intéresse est le premier du recueil et occupe neuf feuillets écrits soit au recto soit au verso. 
On trouve une allusions à ce document dans des ouvrages parus en 1584 et 1606 . Dans celui de 1606, Elogi historici di alcuni personaggi della Famiglia Castiglione, par Antonio Beffa Negrini, la Lettre est justement rappelée au début du registre des lettres de Baldassar.

Le document diffère de l'autographe de Baldassar Castiglione par des erreurs de copie, par le manque de la liste des monuments détruits, et par la formule de politesse ajoutée qui, en revanche, le lie à l'édition de Padoue de 1733.

Je pense que le manuscrit entier, à savoir les presque 162 lettres, est celui que l'homme de lettres de Mantoue, Bernardino Marliani - qui modifie son premier recueil (conservé maintenant aux Archives de Turin) en diminuant les lettres «politiques» de Baldassar (concernant les Montefeltros, les Gonzague et la Papauté) et en augmentant les lettres privées (celles adressées d'Espagne à sa mère Aloisia, par exemple) avait préparé pour la publication dans les années soixante-dix et quatre-vingt du XVI siècle, et qui est demeuré un projet inachevé. Le projet avait été suggéré par le comte Camillo Castiglione, le fils aîné de Baldassar, pour conférer à son père une réhabilitation morale et politique, puisque, comme on le sait, le pape Clément VII avait rejeté sur ce personnage la responsabilité du Sac de Rome de 1527.

\section{Bibliographie}

F. P. Di Teodoro, «Vitruvio, Piero della Francesca, Raffaello : note sulla teoria del disegno d'architettura nel Rinascimento », Annali di architettura, 14 (2002), p. 35-54.

Id., Raffaello, Baldassar Castiglione e la Lettera a Leone X, Con l'aggiunta di due saggi raffaelleschi, presentazione di $\mathrm{C}$. Thoenes, presentazione alla prima edizione di M. Dalai Emiliani, Bologne, Minerva Edizioni, 2003 ( $2^{\mathrm{e}}$ éd.).

Raphaël et Baldassar Castiglione, La Lettre à Léon X, F. P. Di Teodoro (éd.), Françoise Choay et Michel Paoli (trad.), Paris, Les Éditions de l’Imprimeur, 2005. 